\title{
MATURATION AND FERTILISATION OF SHEEP OOCYTES CULTURED IN SERUM-FREE MEDIUM CONTAINING SILK PROTEIN SERICIN
}

\author{
Cut YASMIN ${ }^{1}$, Takeshige Otol $^{2^{*}}$, Mohamad Agus SeTIADI ${ }^{1}$ \\ and Ni Wayan Kurniani KARJA ${ }^{1}$ \\ ${ }^{1}$ Division of Reproduction, Department of Clinic, Reproduction, and Pathology, Bogor \\ Agricultural University, Bogor, Jawa Barat, Indonesia; ${ }^{2}$ Laboratory of Animal Reproduction, \\ The United Graduate School of Veterinary Science, Yamaguchi 753-8515, Japan
}

(Received 14 June 2014; accepted 29 October 2014)

\begin{abstract}
Sericin is a water-soluble component of silk and has been used as a biomaterial due to its antibacterial and ultraviolet radiation-resistant properties. This study was designed to evaluate the effect of sericin supplementation in a maturation medium on the meiotic competence and fertilisability of sheep oocytes. $\mathrm{Cu}$ mulus-oocyte complexes (COCs) were cultured in TCM199 supplemented with sericin at various concentrations of 0 (control), $0.1,0.25$ and $0.5 \%$, either with or without bovine serum albumin (BSA). When the COCs were matured without BSA, the supplementation of $0.1 \%$ sericin significantly increased the rates of maturation to metaphase II and the total fertilisation of oocytes compared with the other concentrations of sericin. When the COCs were matured with BSA, the beneficial effects of $0.1 \%$ sericin supplementation on the maturation and fertilisation of oocytes were not observed. Our findings indicate that supplementation with $0.1 \%$ sericin during maturation culture may improve the nuclear maturation and fertilisability of sheep oocytes. Moreover, it may be possible to replace BSA with sericin in chemically defined media without the risk of disease transmission.
\end{abstract}

Key words: Albumin, antioxidants, in vitro maturation, meiotic competence, ovine oocyte, silk protein, sericin

Sericin is a protein obtained from silk cocoons (Dash et al., 2008). It is the water-soluble component of silk and is used as a biomaterial because of its antibacterial and UV-resistant properties (Zhang, 2002). It has been shown that sericin has a strong antioxidant activity that suppresses lipid peroxidation and inhibits tyrosinase activity (Kato et al., 1998). Dash et al. (2008) demonstrated that sericin prevented oxidative stress in a skin fibroblast cell line when exposed to hydrogen peroxide and inhibited the production of intracellular hydrogen peroxide in keratinocytes. Our group has recently reported that sericin increased the preimplantation development and quality of bovine embryos produced in vitro

*Corresponding author; E-mail: otoi@yamaguchi-u.ac.jp; Phone/Fax: 0081 (83) 933-5904 
and reduced the oxidative stress exerted by hydrogen peroxide on embryos in culture (Isobe et al., 2012).

Serum that includes bovine serum albumin (BSA) is routinely added to the culture medium for the in vitro maturation of sheep oocytes (de Matos et al., 2002; Souza-Fabjan et al., 2013). The addition of serum to the culture medium can carry pathogens, such as bovine spongiform encephalopathy (BSE) prions and viruses, and it can increase the risk of disease transmission (Thibier, 2006). For these reasons, there has been a trend to use more defined or at least semidefined maturation media. In this study, the meiotic competence and fertilisability of sheep oocytes matured in serum-free medium using sericin were evaluated.

\section{Materials and methods}

\section{Sericin}

Pure Sericin ${ }^{\mathrm{TM}}$ was purchased from Wako Pure Chem. Industries, Ltd. (Osaka, Japan). The molecular weight of Pure Sericin is approximately $33 \mathrm{kDa}$, as sericin is hydrolysed during the purification of this product. The amino acid composition of the sericin used in this study was as follows: Ser, 34.2; Asp, 16.4; Gly, 14.8; Thr, 8.2; Arg, 6.1; Ala, 4.6; Glu, 3.8; Val, 3.6; Tyr, 3.1; Lys, 1.9; His, 1.8; Leu, 1.0; I-leu, 0.5; Pro and Ph-ala, < 0.05 (mol\%).

\section{Oocyte collection and in vitro maturation (IVM)}

Sheep ovaries were obtained from an abattoir and transported to the laboratory in $0.9 \%$ physiological saline supplemented with antibiotics $[100 \mathrm{IU} / \mathrm{ml}$ penicillin and $0.1 \mathrm{mg} / \mathrm{mL}$ streptomycin (Sigma-Aldrich, St. Louis, MO, USA)] at $35^{\circ} \mathrm{C}$. Each ovary was sliced repeatedly with a scalpel blade to release cumulusoocyte complexes (COCs) into a dish containing phosphate buffered saline (PBS) supplemented with $0.3 \%$ bovine serum albumin (BSA; Sigma-Aldrich), and antibiotics. Only oocytes with a uniform ooplasm and compact cumulus cells were used in this experiment. The COCs were cultured in a maturation medium, in which the basic medium consisted of 25 mM HEPES tissue culture medium 199 with Earle's salts (TCM199; Invitrogen Co., Carlsbad, CA, USA), supplemented with $2 \mathrm{IU} / \mathrm{mL}$ human chorionic gonadotropin (Kyoritsu Seiyaku, Tokyo, Japan), $2 \mathrm{IU} / \mathrm{mL}$ pregnant mare serum gonadotropin (Kyoritsu Seiyaku), and $50 \mu \mathrm{g} / \mathrm{mL}$ gentamicin (Sigma-Aldrich). The maturation medium was equilibrated for $2 \mathrm{~h}$ in a $\mathrm{CO}_{2}$ incubator before use in the culture. Oocyte maturation was performed under mineral oil (Sigma-Aldrich) by culturing approximately $10-15$ COCs in $100-\mu \mathrm{L}$ droplets in Petri dishes. All dishes were incubated at $39{ }^{\circ} \mathrm{C}$ in a humidified atmosphere of $5 \% \mathrm{CO}_{2}$ for $24 \mathrm{~h}$. 
After the maturation culture, oocytes were denuded from the cumulus cells by repeated pipetting in PBS containing $0.25 \%$ hyaluronidase (Sigma-Aldrich). Denuded oocytes were then washed and mounted on a slide and overlaid with a cover slip supported by Vaseline stripes: paraffin mixture $(9: 1)$. Oocytes were placed in an acetic acid: methanol fixative $(1: 3 \mathrm{v} / \mathrm{v})$ for a minimum of $48-72 \mathrm{~h}$ and then were stained with aceto-orcein ( $2 \%$ orcein and $45 \%$ acetic acid) and rinsed with acetic acid. Stained oocytes were examined under a phase-contrast microscope and classified according to their stage in the maturation process as germinal vesicle $(\mathrm{GV})$, germinal vesicle breakdown (GVBD), metaphase I (MI), or metaphase II (MII).

\section{In vitro fertilisation (IVF)}

Frozen-thawed spermatozoa were prepared and used for fertilisation. Frozen sperm were thawed at $30-32{ }^{\circ} \mathrm{C}$ for $30 \mathrm{sec}$ and were diluted in IVF media that consisted of $90 \mathrm{mM} \mathrm{NaCl}, 12 \mathrm{mM} \mathrm{KCl}, 25 \mathrm{mM} \mathrm{NaHCO}, 0.5 \mathrm{mM} \mathrm{NaH}_{2} \mathrm{PO}_{4}$, $0.5 \mathrm{mM} \mathrm{MgSO}, 10 \mathrm{mM}$ sodium lactate, $3 \mathrm{mg} / \mathrm{mL}$ BSA, $5 \mathrm{mM}$ caffeine, and $50 \mu \mathrm{g} / \mathrm{mL}$ gentamicin. The sperm suspension was centrifuged at $500 \times \mathrm{g}$ for 5 min. After centrifugation, a portion of the sperm pellet was diluted in IVF media. The sperm concentration was adjusted to $1 \times 10^{6}$ spermatozoa $/ \mathrm{mL}$. COCs (10-15 oocytes) were introduced into $100 \mu 1$ of the sperm microdroplet and then co-incubated at $39{ }^{\circ} \mathrm{C}$ in a humidified atmosphere of $5 \% \mathrm{CO}_{2}$ for $14 \mathrm{~h}$.

After $14 \mathrm{~h}$ of co-incubation, the oocytes were fixed to assess their fertilisation as described above. Presumed denuded zygotes were mounted on glass slides, fixed, stained with aceto-orcein, and examined under a phase-contrast microscope. Oocytes containing both female and male pronuclei were considered fertilised and were categorised as monospermic or polyspermic according to the quantities of swollen sperm heads and pronuclei in the cytoplasm.

\section{Experimental design}

In the first experiment, COCs (86 oocytes per group) were cultured in the maturation medium supplemented with sericin at various concentrations of 0 (control), $0.1,0.25$ and $0.5 \%$, either with or without BSA supplementation, to evaluate the effects of sericin supplementation during a maturation culture on the meiotic competence of sheep oocytes. The nuclear status of the oocytes was examined after maturation culture for $24 \mathrm{~h}$, as described above.

In the second experiment, we evaluated the effects of sericin supplementation during maturation culture on the fertilisation of sheep oocytes cultured with or without BSA. In the first experiment, we found that the maturation rate of oocytes decreased when the maturation medium was supplemented with $0.5 \%$ sericin. Therefore, this sericin concentration $(0.5 \%)$ was excluded in this experiment. The COCs ( 85 oocytes per group) were cultured for $24 \mathrm{~h}$ in the maturation 
medium supplemented with sericin at various concentrations of 0 (control), 0.1 and $0.25 \%$, either with or without BSA supplementation. After the maturation culture, the oocytes were co-incubated with spermatozoa for $14 \mathrm{~h}$ and then fixed to evaluate fertilisation.

\section{Statistical analysis}

All experiments were repeated nine or ten times. The mean values of maturation and fertilisation of the oocytes were analysed by analysis of variance (ANOVA) using the general linear models (GLM) procedure in SAS (SAS for Windows, version 9.1, SAS Institute Japan, Tokyo, Japan). The statistical model included BSA supplementation, concentration of sericin, and two-way interactions. Differences with a probability value of $\mathrm{P} \leq 0.05$ were considered to be statistically significant.

\section{Results}

In the first experiment, when evaluating the maturation rates after culture for $24 \mathrm{~h}$, a significant interaction between BSA supplementation and the concentration of sericin was observed $(\mathrm{P}<0.01)$. When the COCs were matured without BSA, the supplementation of sericin increased the maturation rates of oocytes compared with the control oocytes cultured without sericin, irrespective of the concentration of sericin used (Fig. 1). Moreover, the maturation rate of oocytes cultured with $0.1 \%$ sericin $(83.7 \%)$ was significantly higher $(\mathrm{P}<0.01)$ than that of oocytes cultured with 0.25 and $0.5 \%$ sericin $(63.6 \%$ and $39.7 \%$, respectively). When the COCs were matured with BSA, the maturation rate of the oocytes cultured with $0.1 \%$ sericin $(73.0 \%)$ was similar to that of the control oocytes cultured without sericin $(69.9 \%)$. In contrast, the maturation rates of the oocytes cultured with 0.25 and $0.5 \%$ sericin $(58.1 \%$ and $46.0 \%$, respectively) were significantly lower than those of the control oocytes $(\mathrm{P}<0.05)$.

In the second experiment, when evaluating the total and monospermic fertilisation rates after $14 \mathrm{~h}$ of co-incubation with spermatozoa, a significant interaction between BSA supplementation and the concentration of sericin was observed in the total fertilisation rates $(\mathrm{P}<0.01)$ but not in the monospermic fertilisation rates (Fig. 2). When the COCs were matured without BSA and then fertilised with spermatozoa, the total fertilisation rates of the oocytes matured with $0.1 \%$ sericin were significantly higher $(\mathrm{P}<0.01)$ than those of the oocytes matured with $0.25 \%$ sericin and without sericin $(84.8 \%$ vs. $54.8 \%$ and $16.5 \%$, respectively). When the COCs were matured with BSA, there were no differences in the total fertilisation rates among the groups. Moreover, there were no significant differences in the rates of monospermic fertilisation of the oocytes among the groups, irrespective of sericin and BSA supplementation during maturation culture. 


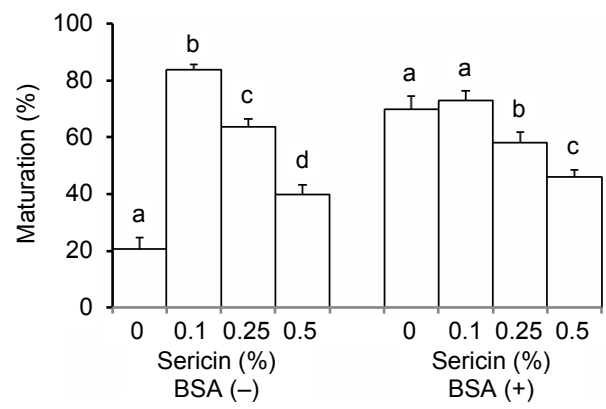

Fig. 1. The effects of sericin supplementation in the maturation medium on the maturation rates of sheep cumulus-oocyte complexes (COCs) cultured with (BSA+) or without BSA (BSA-). The COCs ( 86 oocytes per group) were cultured in a maturation medium supplemented with $0 \%, 0.1 \%$, $0.25 \%$ and $0.5 \%$ of sericin, either with or without BSA supplementation. The bars with disparate letters for each BSA supplementation differ significantly $(\mathrm{P}<0.05)$
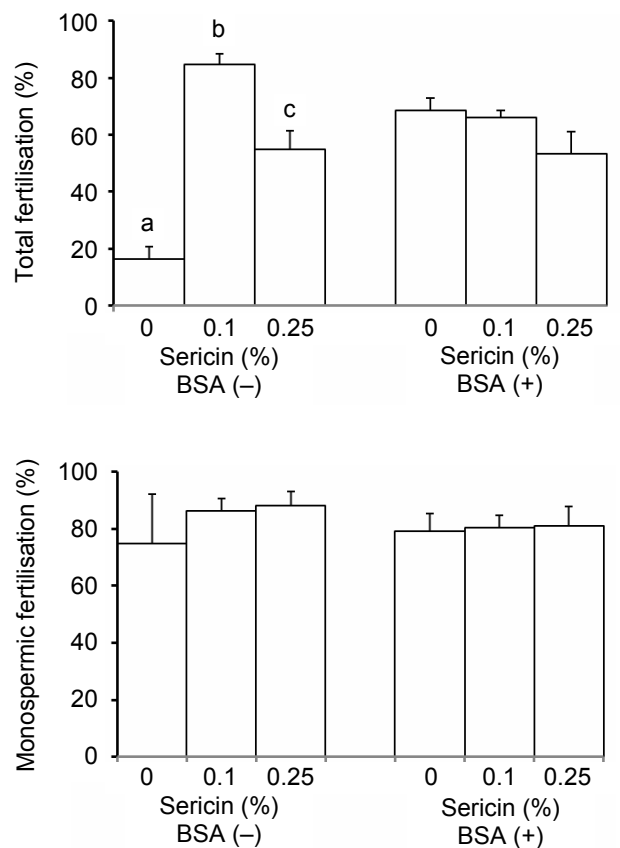

Fig. 2. The effects of sericin supplementation in the maturation medium on the rates of total (A) and monospermic (B) fertilisation of sheep cumulus-oocyte complexes (COCs) cultured with $(\mathrm{BSA}+)$ or without BSA (BSA-). The COCs ( 85 oocytes per group) were cultured in a maturation medium supplemented with $0 \%, 0.1 \%$ and $0.25 \%$ of sericin, either with or without BSA supplementation. The monospermic fertilisation rates were calculated by dividing the numbers of monospermic fertilised oocytes by the total number of fertilised oocytes. The bars with disparate letters differ significantly $(\mathrm{P}<0.05)$ 


\section{Discussion}

Our findings indicate that $0.1 \%$ sericin supplementation can improve the rates of maturation and total fertilisation of sheep oocytes cultured in a maturation medium supplemented without BSA. To the best of our knowledge, the current study is the first to report the effects of sericin supplementation during maturation culture on the meiotic competence of sheep oocytes. Sericin has been reported to stimulate the proliferation of mammalian cells (Terada et al., 2002). Moreover, sericin decreases the level of reactive oxygen species in cultures by preventing $\mathrm{H}_{2} \mathrm{O}_{2}$-induced oxidative stress (Dash et al., 2008), and the mechanism of this antioxidant action is the chelation of trace elements stemming from the remarkably high content of hydroxy amino acids in sericin (Kato et al., 1998). In a previous study, we reported that supplementation with $1.0 \%$ sericin during maturation culture improved the meiotic competence of porcine oocytes and the quality of blastocysts as determined by the DNA fragmentation index (Do et al., 2014). Moreover, we confirmed that sericin can prevent oxidative stress during bovine embryo culture, resulting in the improvement of embryo quality and increased embryonic development (Isobe et al., 2012). Therefore, due to its unique antioxidant potential, sericin might improve the meiotic competence of sheep oocytes by preventing oxidative stress during maturation culture.

On the other hand, when BSA was supplemented in the maturation medium, the beneficial effects of sericin supplementation on the maturation and fertilisation of oocytes were not observed. It has been suggested that BSA can bind many low-molecular-weight compounds of varied origins, including heavy metal ions, free radicals, citrate and steroids (George et al., 2008). Particularly, serum albumin exerts important antioxidant activities in which the molecule acts through its multiple binding sites and free radical-trapping properties (Roche et al., 2008). Our findings may indicate that sericin has the same target as BSA, and thus, the combination of sericin and BSA may overlap. Therefore, the beneficial effects of sericin on the maturation and fertilisation of sheep oocytes might disappear in the presence of BSA.

BSA used for the in vitro maturation of sheep oocytes has sanitary risks, although these are much lower than the risks associated with serum. Sericin is known to be safe and can be added to drugs, over-the-counter drugs, cosmetics, foods and health foods (Kundu et al., 2008). In the present study, when the effects of sericin supplementation in a BSA-free medium were evaluated by a comparison with BSA-containing medium without sericin, the rates of maturation and total fertilisation of oocytes matured in the BSA-free medium with $0.1 \%$ sericin were significantly higher than those of oocytes matured in a BSA-containing medium without sericin $(83.7 \%$ vs. $69.9 \%$ and $84.8 \%$ vs. $68.7 \%$, respectively, $\mathrm{P}<0.05$ ). These results may support the experimental findings of Hosoe et al. (2014) who reported that the rates of nuclear maturation, fertilisation and blasto- 
cyst formation of bovine oocytes matured with sericin were similar to those of oocytes matured with fetal bovine serum. Therefore, sericin is not only suitable for use in the maturation culture of sheep oocytes but is also beneficial for the elimination of a potential source of biological contamination by serum or BSA.

In conclusion, our findings indicate that supplementation of $0.1 \%$ sericin in a maturation culture improves the meiotic competence and fertilisability of sheep oocytes matured in BSA-free medium. However, the beneficial effects of sericin disappear in oocytes matured in a BSA-containing medium. Further studies are necessary to evaluate not only the exact role and mechanism of sericin in sheep oocyte maturation but also the embryonic development after IVF.

\section{Acknowledgement}

This work was partly supported by the JSPS/DGHE Joint Research Project 20112013

\section{References}

Dash, R., Acharya, C., Bindu, P. C. and Kundu, S. C. (2008): Antioxidant potential of silk protein sericin against hydrogen peroxide-induced oxidative stress in skin fibroblasts. BMB Rep. 41, 236-241.

de Matos, D. G., Gasparrini, B., Pasqualini, S. R. and Thompson, J. G. (2002): Effect of glutathione synthesis stimulation during in vitro maturation of ovine oocytes on embryo development and intracellular peroxide content. Theriogenology 57, 1443-1451.

Do, L. T., Namula, Z., Luu, V. V., Sato, Y., Taniguchi, M., Isobe, T., Kikuchi, K. and Otoi, T. (2014): Effect of sericin supplementation during in vitro maturation on the maturation, fertilization and development of porcine oocytes. Reprod. Domest. Anim. 49, e17-20.

George, F., Daniaux, C., Genicot, G., Verhaeghe, B., Lambert, P. and Donnay, I. (2008): Set up of a serum-free culture system for bovine embryos: embryo development and quality before and after transient transfer. Theriogenology 69, 612-623.

Hosoe, M., Yoshida, N., Hashiyada, Y., Teramoto, H., Takahashi, T. and Niimura, S. (2014): Sericin accelerates the production of hyaluronan and decreases the incidence of polyspermy fertilization in bovine oocytes during in vitro maturation. J. Reprod. Dev. 60, 268-273.

Isobe, T., Ikebata, Y., Onitsuka, T., Wittayarat, M., Sato, Y., Taniguchi, M. and Otoi, T. (2012): Effect of sericin on preimplantation development of bovine embryos cultured individually. Theriogenology 78, 747-752.

Kato, N., Sato, S., Yamanaka, A., Yamada, H., Fuwa, N. and Nomura, M. (1998): Silk protein, sericin, inhibits lipid peroxidation and tyrosinase activity. Biosci. Biotechnol. Biochem. 62, 145-147.

Kundu, S. C., Dash, B. C., Dash, R. and Kaplan, D. L. (2008): Natural protective glue protein, sericin bioengineered by silkworms: Potential for biomedical and biotechnological applications. Prog. Polym. Sci. 33, 998-1012.

Roche, M., Rondeau, P., Singh, N. R., Tarnus, E. and Bourdon, E. (2008): The antioxidant properties of serum albumin. FEBS Lett. 582, 1783-1787.

Souza-Fabjan, J. M., Pereira, A. F., Melo, C. H., Sanchez, D. J., Oba, E., Mermillod, P., Melo, L. M., Teixeira, D. I. and Freitas, V. J. (2013): Assessment of the reproductive parameters, 
laparoscopic oocyte recovery and the first embryos produced in vitro from endangered Caninde goats (Capra hircus). Reprod. Biol. 13, 325-332.

Terada, S., Nishimura, T., Sasaki, M., Yamada, H. and Miki, M. (2002): Sericin, a protein derived from silkworms, accelerates the proliferation of several mammalian cell lines including a hybridoma. Cytotechnology 40, 3-12.

Thibier, M. (2006): Biosecurity and the various types of embryos transferred. Reprod. Domest. Anim. 41, 260-267.

Zhang, Y. Q. (2002): Applications of natural silk protein sericin in biomaterials. Biotechnol. Adv. 20, $91-100$ 